\title{
AUTOMORPHISMS OF THE FUNDAMENTAL GROUP OF A CLOSED, ORIENTABLE 2-MANIFOLD ${ }^{1}$
}

\author{
JOAN S. BIRMAN
}

In the following pages we note a relationship which exists between the mapping class group (or homeotopy group) of a closed, orientable 2-manifold and Artin's braid group. Let $T_{o}$ be a closed, orientable 2-manifold of genus $g$, and let $\pi_{1} T_{\theta}$ be its fundamental group. Let $M\left(T_{0}\right)$ be the mapping class group of $T_{0}$, that is the group of all isotopy classes in the space of all orientation-preserving homeomorphisms of $T_{g} \rightarrow T_{g}$, or equivalently [4] the group Aut $\pi_{1} T_{o} / \operatorname{Inn} \pi_{1} T_{g}$. Let $B_{n}$ be the Artin braid group, that is the abstract group on $n-1$ generators $\sigma_{1}, \cdots, \sigma_{n-1}$ with defining relations

$$
\begin{aligned}
\sigma_{i} & \leftrightarrows \sigma_{j}, \quad i=1,2, \cdots, n-1, \quad|i-j| \geqq 2, \\
\sigma_{i} \sigma_{i+1} \sigma_{i} & =\sigma_{i+1} \sigma_{i} \sigma_{i+1}, \quad i=1,2, \cdots, n-2 .
\end{aligned}
$$

The group $M\left(T_{1}\right)$ is the modular group, which is a homomorphic image of $B_{3}$ and also (with an appropriate choice of generators) of $B_{4}$. It was shown by Bergau and Mennicke [1] that $M\left(T_{2}\right)$ is a homomorphic image of $B_{6}$. We are able to generalize these known results to show that $M\left(T_{g}\right)$ contains a sequence of $(n-1)$-generator subgroups which are homomorphic images of $B_{n}$ for $n=3,4, \cdots, 2 g+2$, the union of these subgroups generating all of $M\left(T_{0}\right)$. If $g>2$, all of these subgroups are proper in $M\left(T_{0}\right)$.

Proof. Generators for $M\left(T_{g}\right)$ are known, and we use a particularly simple set which was discovered by M. Dehn [2] and recently rediscovered and simplified by W. B. R. Lickorish [3]. Let $A$ be an annulus in the Euclidean plane, parametrized by $(r, \theta)$, where
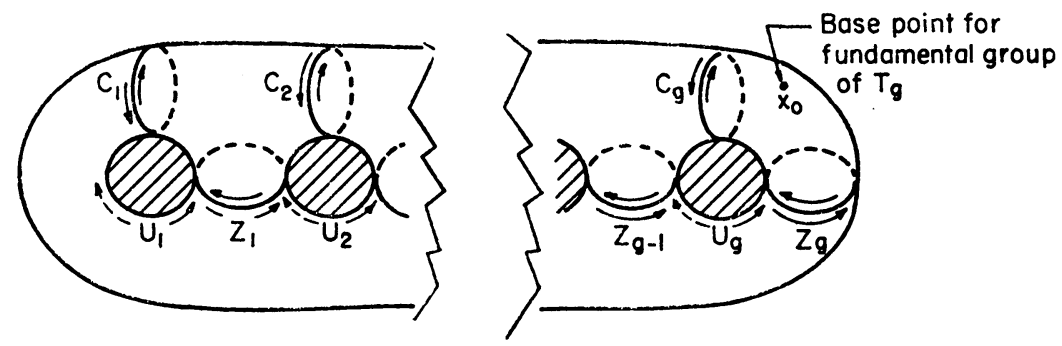

FIGURE 1

Received by the editors May 30, 1968.

1 Work supported in part under Grant GP-5091 of the National Science Foundation. 
$1 \leqq r \leqq 2$ and $\theta$ is a real number $\bmod 2 \pi$. We define a homeomorphism $h: A \rightarrow A$ by $h(r, \theta)=(r, \theta-2 \pi r)$. If $C$ is now any simple closed curve on $T_{g}$, and $e_{C}: A \rightarrow T$, an imbedding of $A \rightarrow$ a neighborhood of $C$ in $T_{g}$, which maps $r=1.5$ onto $C$, then $e_{c} h e_{C}^{-1} / e_{C} A$ may be extended by the identity map on $\left(T_{0}-e_{C} A\right)$ to a homeomorphism $h_{C}: T_{0} \rightarrow T_{0}$, which is called a twist about $C$. It was shown in [3] that the twists $\left\{h_{U_{i}}, h_{Z_{i}}, h_{C_{j}} ; 1 \leqq i \leqq g, 1 \leqq j \leqq g-1\right\}$ about the curves $\left\{U_{i}, Z_{i}, C_{j}\right\}$ in Figure 1 generate $M\left(T_{\theta}\right)$. The arrows indicate the direction of relative motion of points on $T_{0}$ when each such twist is performed.

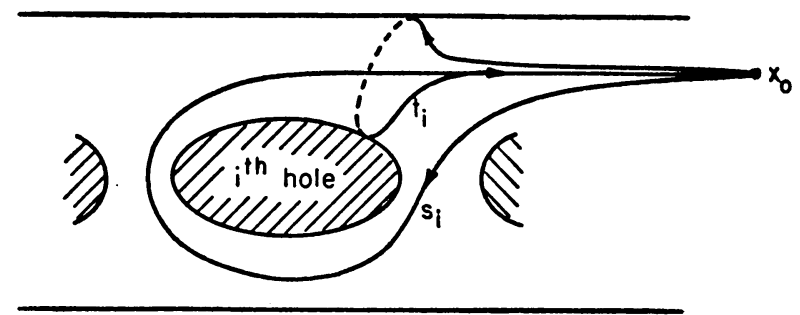

FIGURE 2

Each of these twists induces an automorphism of $\pi_{1} T_{g}$, and these can be determined explicitly as follows. We take a base point for $\pi_{1} T_{g}$ as indicated in Figure 1 , and define generators $\left\{t_{i}, s_{i} ; i=1,2\right.$, $\cdots, g\}$ for $\pi_{1}\left(T_{0}, x_{0}\right)$ which are represented by the loops illustrated in Figure 2. In terms of these generators the group Aut $\pi_{1} T_{0}$ has the single defining relation

$$
\prod_{i=1}^{0} s_{i}^{-1} \tilde{t}_{i} s_{i} \tilde{t}_{i}^{-1}=1
$$

where

$$
\tilde{t}_{1}=s_{1} t_{1} s_{1}^{-1}, \tilde{t}_{i}=s_{i}\left(\tilde{t}_{i-1} \bar{t}_{i-1}^{-1} t_{i}\right) s_{i}^{-1} \text { if } i \neq 1 .
$$

The automorphism induced by a typical twist $h_{z_{g-1}}$ on the $t_{i}, s_{i}$ will now be determined. Clearly $h_{z_{0-1}}$ will not effect any of the $t_{i}$, nor will
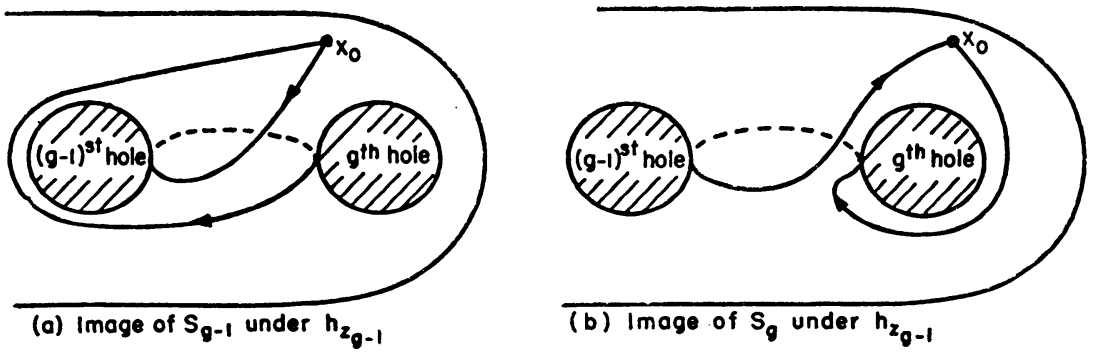

Figure 3 
$h_{z_{0-1}}$ effect $s_{i}$ if $i \neq g$ or $g-1$. To determine the effect of $h_{z_{0-1}}$ on $s_{\theta-1}$ and $s_{g}$, we observe that, under the action of $h_{z_{g-1}}: s_{g-1}$ and $s_{0}$ will be altered to the curves illustrated in Figures $3 \mathrm{a}$ and $3 \mathrm{~b}$ respectively, which can be seen to be homotopic to the curves $t_{0-1}^{-1} t_{0} s_{0-1}$ and $s_{0} t_{0}^{-1} t_{0-1}$ on $T_{g}$. The induced automorphisms for all possible cases can be determined from similar pictures, and are found to be:

$$
\begin{aligned}
h_{U_{i}}: t_{i} & \rightarrow t_{i} s_{i} \\
h_{C_{i}}: s_{j} & \rightarrow t_{i} s_{j} t_{i}^{-1} \quad \text { if } j<i \\
t_{j} & \rightarrow t_{i} t_{j} t_{i}^{-1} \quad \text { if } j<i \\
s_{i} & \rightarrow s_{i} t_{i}^{-1} \\
h_{Z_{i}}: s_{i} & \rightarrow t_{i}^{-1} t_{i+1} s_{i} \\
s_{i+1} & \rightarrow s_{i+1} t_{i+1}^{-1} t_{i} \quad \text { if } i=1,2, \cdots, g-1 \\
h_{Z_{g}}: s_{g} & \rightarrow t_{g}^{-1} s_{g}
\end{aligned}
$$

where it is understood that every generator which is not listed explicitly is unaltered by the $h$ 's.

Since $\left\{h_{U_{i}}, h_{Z_{i}}, h_{C j} ; 1 \leqq i \leqq g, 1 \leqq j \leqq g-1\right\}$ generate $M\left(T_{g}\right)$, the automorphisms listed above generate Aut $\pi_{1} T_{g} / \operatorname{Inn} \pi_{1} T_{g}$. This provides a very simple tool for verifying relations in $M\left(T_{g}\right)$ : one simply calculates the automorphism induced by a word one suspects is a relator, and observes whether this automorphism is an inner automorphism of $\pi_{1} T_{g}$. Using this procedure, we find the following relations hold among the $h_{U_{i}}, h_{Z_{i}}, h_{C_{j}}$.

(1) $h_{U_{i}} \leftrightarrows h_{U_{j}}, \quad i \neq j$,

(2) $h_{C_{i}} \leftrightarrows h_{C_{j}}, \quad i \neq j$

(3) $h_{Z_{i}} \leftrightarrows h_{Z_{j}}, \quad i \neq j$,

(4) $h_{U_{i}} \leftrightarrows h_{C_{j}}, \quad i \neq j$

(5) $h_{z_{i}} \leftrightarrows h_{C_{j}}, \quad i, j=1,2, \cdots, g$

(6) $h_{U_{i}} \leftrightarrows h_{Z_{j}}, \quad i \neq j-1$ or $j$,

(7) $h_{U_{i}} h_{Z_{i}} h_{U_{i}}=h_{Z_{i}} h_{U_{i}} h_{Z_{i}}, \quad i=1,2, \cdots, g$,

(8) $h_{U_{i+1}} h_{Z_{i}} h_{U_{i+1}}=h_{Z_{i}} h_{U_{i+1}} h_{Z_{i}}, \quad i=1,2, \cdots, g-1$,

(9) $h_{C_{i}} h_{U_{i}} h_{C_{i}}=h_{U_{i}} h_{C_{i}} h_{U_{i}}, \quad i=1,2, \cdots, g$,

(10) $\left(h_{C_{1}} h_{U_{1}} h_{Z_{1}} h_{U_{2}} h_{Z_{2}} \cdots h_{U_{\theta}} h_{Z_{\vartheta}}\right)^{2 \theta+2}=1$,

(11) $\left(h_{C_{1}} h_{U_{1}} h_{Z_{1}} h_{U_{2}} h_{Z_{2}} \cdots h_{U_{g}} h_{Z_{9}} h_{U_{0}} \cdots h_{Z_{2}} h_{U_{2}} h_{Z_{1}} h_{U_{1}} h_{C_{1}}\right)^{2}=1$.

We cannot say whether this system is complete.

It then follows that there is a homomorphism $\phi: B_{20+2} \rightarrow M\left(T_{\theta}\right)$ defined by $\phi\left(\sigma_{1}\right)=h_{C_{1}}, \phi\left(\sigma_{2 j}\right)=h_{U_{j}}, \phi\left(\sigma_{2 j+1}\right)=h_{z_{j}}$, for $j=1,2, \cdots, g$. 
Moreover, since the center of $B_{n}$ is known to be. infinite cyclic and generated by $\left(\sigma_{1} \sigma_{2} \cdots \sigma_{n-1}\right)^{n}$, in view of relation (10) we have that the center of $B_{20+2} \subset \operatorname{ker} \phi$. This is not, however, the full kernel because relation (11) also holds in $M\left(T_{\vartheta}\right)$, and from previous studies of the group $B_{n}$ it is known that relation (11) is not a consequence of the others in $B_{n} /$ center.

Moreover, the $(2 g-2 i+3)$-generator subgroups of $M\left(T_{\vartheta}\right)$ generated by $\left\{h_{C_{i}}, h_{U_{i}}, h_{Z_{i}}, h_{U_{i+1}}, h_{Z_{i+1}}, \cdots, h_{U_{q}}, h_{Z_{q}}\right\}$ are homomorphic images of $B_{2 g-2 i+4}$ for each $i=2, \cdots, g$ under the homomorphism $\phi_{i}: B_{2 g-2 i+4} \rightarrow M\left(T_{0}\right)$ defined by $\phi_{i}\left(\sigma_{1}\right)=h_{C_{i}}, \phi_{i}\left(\sigma_{2 j}\right)=h_{U_{j+i-1}}, \phi_{i}\left(\sigma_{2 j+1}\right)$ $=h_{z_{j+i-1}}$ for $j=1,2, \cdots, g-i+1$.

Finally, the $(2 g-2 i+2)$-generator subgroups of $M\left(T_{o}\right)$ which are generated by $\left\{h_{U_{i}}, h_{Z_{i}}, h_{U_{i+1}}, h_{Z_{i+1}}, \cdots, h_{z_{g}}\right\}$ are homomorphic images of $B_{2 g-2 i+3}$ for each $i=1,2, \cdots, g$ under the homomorphism $\psi_{i}$ defined by $\psi_{i}\left(\sigma_{2 j-1}\right)=h_{U_{j+i-1}}, \psi_{i}\left(\sigma_{2 j}\right)=h_{z_{j+i-1}}$ for $j=1,2, \cdots$, $g-i+1$.

\section{REFERENCES}

1. P. Bergau and J. Mennicke, Über topologische Abbildungen der Brezelfläche vom Geschlecht 2, Math. Z. 74 (1960), 414-435.

2. M. Dehn, Die Gruppe der Abbildungsklassen, Acta Math. 69 (1938), 135-206.

3. W. B. R. Lickorish, $A$ finite set of generators for the homeotopy group of a 2 manifold, Proc. Cambridge Philos. Soc. 60 (1964), 769-778.

4. Wilhelm Magnus, Abraham Karass and Donald Solitar, Combinatorial group theory, Interscience, New York, 1966, p. 75.

Courant Institute, New York University 